\title{
The Study on the Architecture of Public knowledge Service Platform Based on Collaborative Innovation
}

\author{
Chang ping Hu, Min Zhang, Fei Xiang \\ Center for the Studies of Information Resources of Wuhan University, \\ Wuhan,430072,China, sxzhangm@yahoo.com.cn
}

\begin{abstract}
The knowledge service platform is seen as the vital intermediaries in innovation cycle, and for users to locate, exchange and acquire knowledge in a systematic way. It is increasingly recognizing the powerful role that knowledge service platform provide in building a national innovation capacity. The knowledge service platforms in the China's national innovation system, mostly based on the digital libraries, are supported and constructed by diverse departments and systems. These platforms disperse in construction, have respective division and orientation. However, along with the development of information technology and the revolution of innovation model, the requirements for the reconstruction of knowledge service platform in innovation system are brought forward. In this paper, we analyze the resources integration framework of the existing public service platform, and put forward the architecture of the public knowledge service platform based on collaborative innovation; we also discuss its concrete implementation.
\end{abstract}

\section{Introduction}

The knowledge service platform, an important part of the national innovation system, is seen as the vital intermediaries in innovation cycle, and for users to locate, exchange and acquire knowledge in a systematic way, with a view to development of new products, processes and services. It is increasingly recognizing the powerful role that knowledge service platform provide in building a national innovation capacity[1].The knowledge service platforms in the China's national innovation system, mostly based on the digital libraries, are supported and constructed by diverse departments and systems. These platforms disperse in construction, have respective division and orientation. However, along with the development of information technology and the revolution of innovation model as well as the change

Please use the following format when citing this chapter:

Hu, C. P., Zhang, M., Xiang, F., 2007, in IFIP International Federation for Information Processing, Volume 252, Integration and Innovation Orient to E-Socicty Volume 2, eds. Wang, W., (Boston: Springer), pp. 9-17. 
of international information environment, the development model of knowledge service platform according to the system construction and the department organization receives challenges coming from various aspects, the requirements for the reconstruction of knowledge service platform in innovation system are brought forward.

Given this background, this paper mainly discusses the construction and development strategy of public knowledge service platform based on the collaborative innovation. Section two analyzes the driving mechanism of the innovation service platform and explores the influence of the new innovation paradigm and technology environment. Section three introduces Hubei province Science and Technology information sharing service platform as a case to illustrate the practice of the service sharing platform based on the information integration, and points out its shortage of knowledge collaboration. Section four we set up a framework of public knowledge service platform base on collaborative innovation, expound its construction and implementation. Finally we give some possible avenues for further research.

\section{The driving mechanism of knowledge service platform in national innovation system}

Nowadays, the innovation way has been undergone great changes. The new innovation model springs up continually such as open innovation, online innovation, distributed innovation and connected innovation [2-4]. Innovation development puts greater emphasis on interaction and collaboration of the service platform. The knowledge service platform, as an important intermediary in the whole process of the innovative chain, provides knowledge and information service for innovative system factors (government, educational and research institution, enterprise, etc.), facilitating resource sharing and behavior interaction among the factors and realizing the collaborative innovation effect.

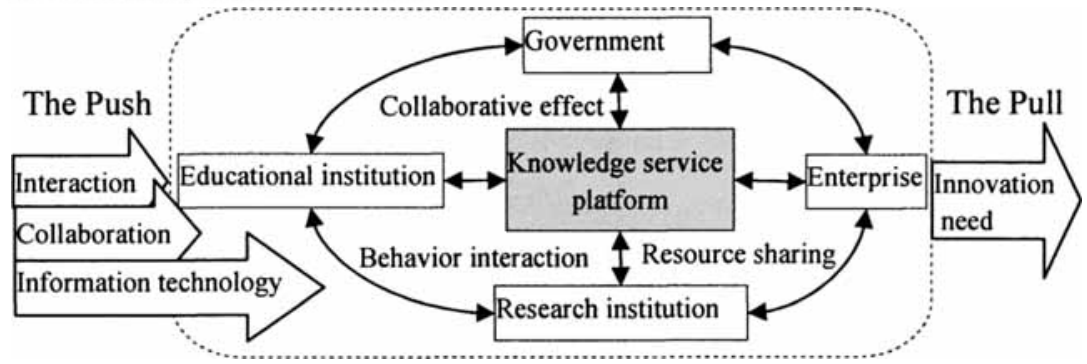

Fig. 1. The driving mechanism of knowledge service platform in national innovation system

- Innovation requires the development of new relationships among and between businesses, government, education and research organizations, workers and society in general. Above all, it requires a culture of collaboration, a symbiotic relationship between research and commercialization [5]. 
- The innovation process relies heavily on groups collaboration across institutions and time zones, sharing data, complementary expertise, ideas, so requires a closer integration among the innovative resource, activities and personnel. At the same time it requires that the service platform can promote coupling and interaction in all links of innovation chain to enhance the whole system innovation ability.

- Under the distributed, heterogeneous and dynamic changes environment, knowledge service platform are aiming on a transition from an integrated, centrally controlled system to a dynamic configurable federation of services and information collections. This transition is inspired by new technology trends and developments, which includes technologies like Web services and the Grid as well as the success of new paradigms like Peer-to-Peer Network and Service oriented Architectures.

\section{Practice of Knowledge service platform based on information resource integration and its shortage of knowledge cooperation}

In recent years, China has constructed the sharing service platform including National Science and Technology Library (NSTL) presided by Ministry of Science and Technology of the People's Republic of China[6], China Academic Library and Information System (CALIS) presided by Ministry of Education[7], regional and national sharing platform of literature and information resource with the National Library of China at the core[8], National Science Library of Chinese Academy of Sciences (NSL)[9], and so on. These platforms promoted national innovation development greatly. Nevertheless, the platforms developed by various sectors work in their own ways and information can't manage in unity. In the course of constructing innovational nation, collaborative innovation emphasizes on closer cooperation and mutual integration, so strategic request of reconstruction of knowledge platform across industries and systems is brought forward.

Hubei Science and Technology information sharing service platform is the service sharing platform across system. It is supported by Hubei Academy of Scientific and Technical Information, which has integrated four information systems including high education, Chinese Academy of Sciences, science and technology information and public libraries[10]. In 2005, Hubei Academy of Scientific and Technical Information realized the integration between education network and science and technology network through the interconnection between Changiiang Technology and Economy Information Network and China Education and Research Network. The successful integration between Changjiang Technology and Economy Information Network and Huazhong center of CALIS (The Library of Wuhan University) realized the retrieval on the unified platform, realized the synchronous transmission of science and technology literature and information through the seamless interconnection project cooperated with NSTL as well(see figure 2). The service pattern of the platform includes regional service station pattern, commissioned service pattern, database service pattern and end-user service pattern. 


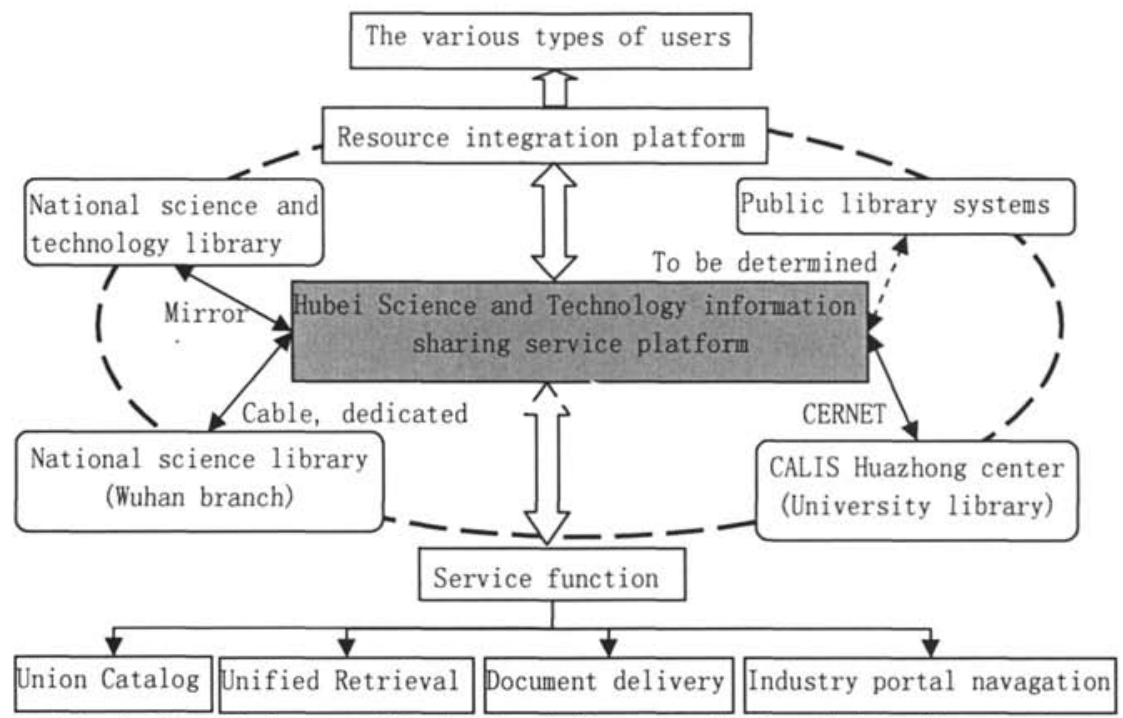

Fig. 2. Hubei Science and Technology information sharing platform framework

Hubei Science and Technology information sharing platform is the typical representative of innovative service platform based on information resource integration, which has already realized resource integration and service sharing across the space-time and systems to some degree. However the existing platform still has inadequate in knowledge collaboration:

- the platform has low efficiency of human resources and can't organize the innovational team according to the task need, so well as support the work of the cooperation team.

- the utilization efficiency of knowledge is not high enough and the research focuses on the information transmission and information management, not on the knowledge, can't promote the increment of knowledge in the cooperation course.

- From the angle of the technology, the present platform is based on the physical interconnection. The resource offering and service sharing are static. It doesn't offer the framework of resource and service sharing among multiorganizations and can't realize the large-scale resource and service sharing. Besides, the cost of the system is high while the efficiency is low.

\section{Construction and Implementation of the knowledge service platform based on the collaborative innovation}

In order to satisfy the demand of the collaborative innovation, As figure 3 shows, we construct the public knowledge service platform, which can be divided into three layers: physical layer, logical layer and user layer. The physical layer mainly consists of various kinds of distributed repositories (including publicly funded digital repositories, Industry Information platforms, business information 
platforms, etc.) .These distributed and heterogeneous digital repositories provide the material for dynamic configuration of services and information collections. On the basis of the physical level, the logical layer realizes the centralized resource allocation and mediation, as well as dynamic deployment service. The user layer provides the knowledge access based on the innovation demand for the different user.

The architecture of Collaboration innovation-oriented public knowledge service platform reflects the following three characteristics:

- The resources choice is decided by the users' innovation demands and the standard which user own defines to;

- Knowledge service platform provide dynamic service through integration of distributed resource;

- The knowledge service process is base on the Virtual organization (VO) mechanism.

Now through the functions and processes of the public knowledge service platform we explain its realization to support collaborative innovation.

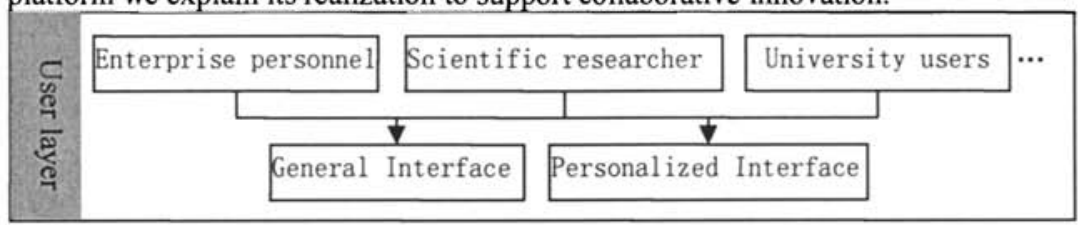

\begin{tabular}{|c|c|c|c|}
\hline \multirow{5}{*}{$\begin{array}{c}5 \\
\frac{5}{\circ} \\
\frac{10}{2} \\
\frac{1}{1}\end{array}$} & Vo's configuration \& management & Knowledge service & $\begin{array}{c}\text { Resource allocation } \\
\text { Management }\end{array}$ \\
\hline & User Registration Service & Knowledge modeling & Resource scheduling \\
\hline & Collaborative development service & Knowledge retrieval & Resource aggregation \\
\hline & Process management service & Knowledge discovery & Resource mining \\
\hline & Service Scheduling management & Knowledge updating & Resource monitor \\
\hline
\end{tabular}

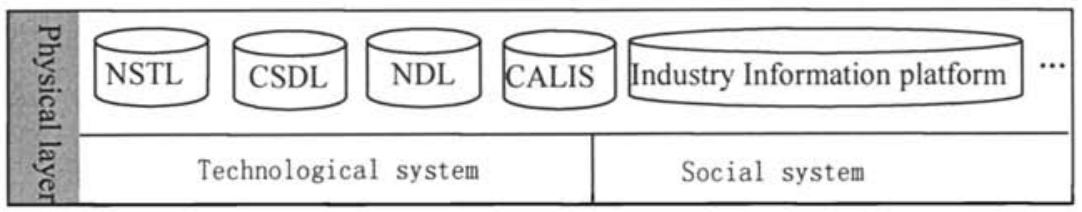

Fig. 3. the Architecture of the Public knowledge service platform based on collaborative innovation

\subsection{VO's creation and execution based on Service-Oriented Architecture}

Following the development of knowledge globalization, the technology innovation across profession and discipline pervades all over the world, Virtual organization (VO) and Virtual enterprises and so on appear one after another. VO integrates the unique advantages of diverse organizations through resources sharing and risk pooling to boost innovation. With providing the VO's configuration and the task management mechanism, the knowledge service platform we constructed will 
facilitate the integration of innovation resources , innovation personnel and innovation activities.

Considering the technical feasibility, We realize the creation of virtual organizations and operations management based on SOA ( Service-Oriented Architecture ) .SOA is a kind of software development architecture suitable for the changing environment, which can carry on the distributional deployment, the combination and the use for the loose coupling application module according to the demand[11-12]. Web service based on SOA describes three roles and three basic operations (sees figure 4). Three roles are service provider, service requester and service broker; three basic operation are publish, find and bind respectively.

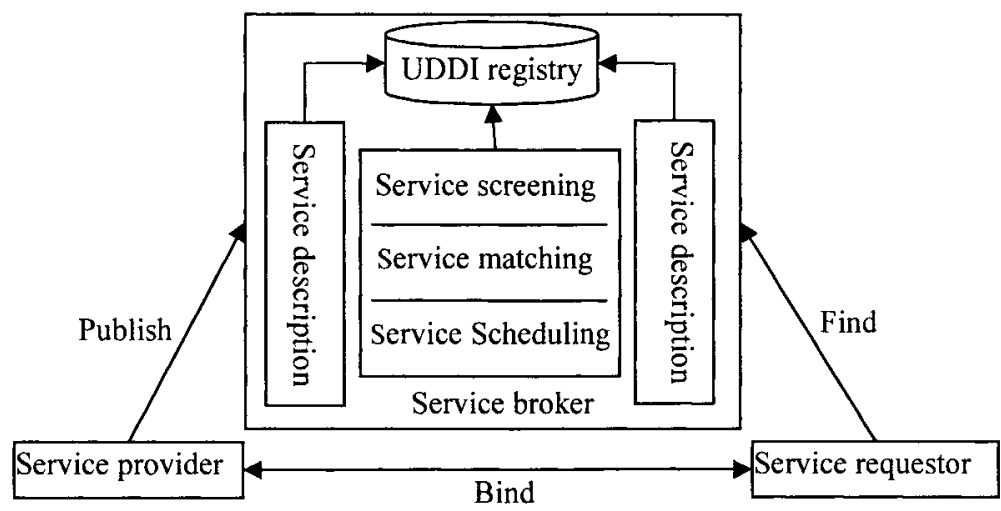

Fig. 4. SOA architecture

Based on SOA architecture, Innovation main bodies release the innovation information through registry module, meanwhile inquiry relevant information which have been registered, and use certain appraisal mechanism to Choose the appropriate partner and set up the virtual organization. The virtual innovation organization integrates several services to complete a specific innovation project. After the completion of the project, registry members cancel their functions, and withdraw from alliance. As a result, the entire dynamic VO declares on the dissolution.

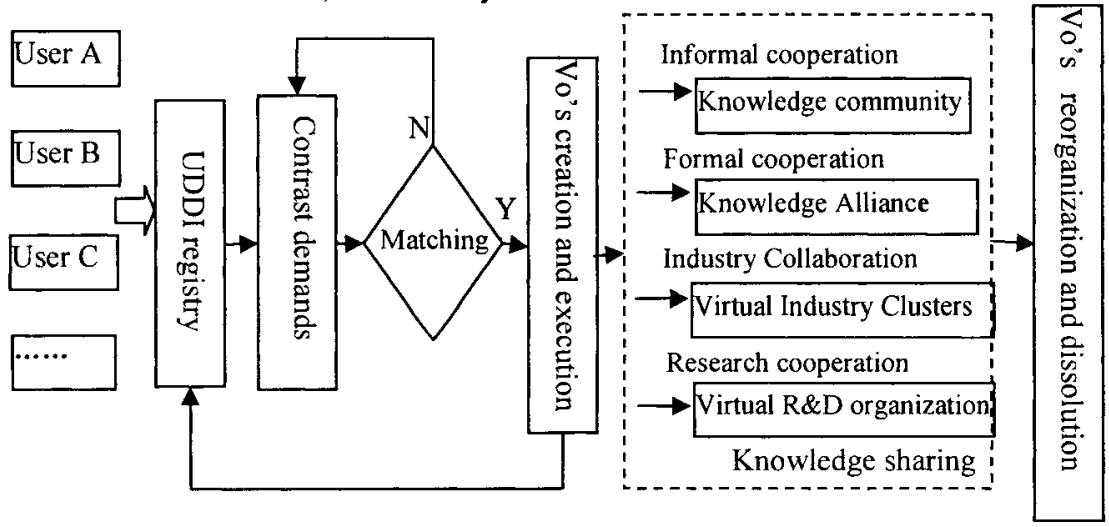


Fig. 5. VO's creation and execution

\section{2 task-driven resource allocation and coordination services}

In the operation of $\mathrm{VO}$, task management module is used to realize the dynamic dispatch and assignment of the innovation project or task, in the mean time manage task operation condition in order to optimize the resource allocation. Decomposes the task and distributes each member of virtual organization, then discoveries the knowledge unit in the light of each business activity, and unifies the knowledge unit and the specific business process so as to establish mapping relations. Extracts the knowledge from distributional repository and completes the process from knowledge representation, organization, retrieval and knowledge innovation.

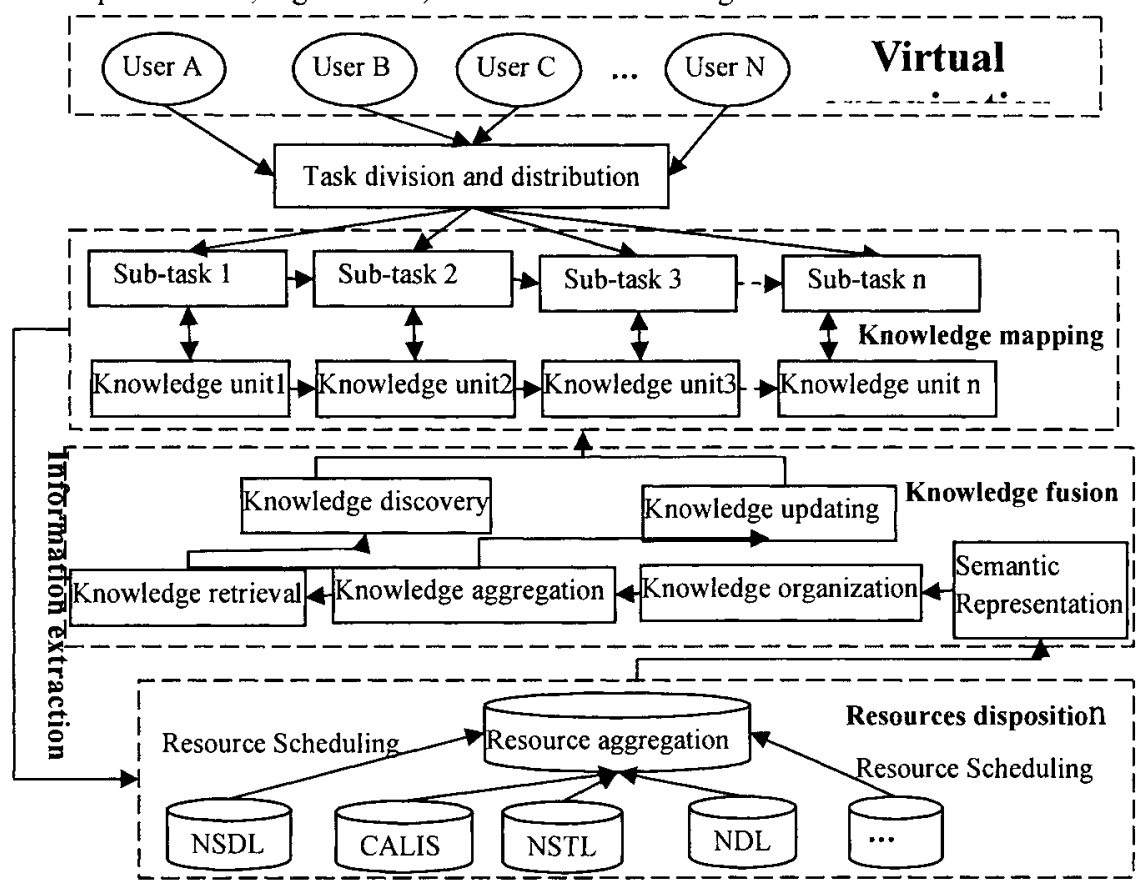

Fig. 6. task-driven process of knowledge service platform

\section{Conclusion}

Although innovation itself is a continuous process for producing new ideas, productions, services and tools, The operation of innovation is highly environmentdependent or platform-dependent. The innovation knowledge must be transferred through the integrated information infrastructure; the consummation of innovation chain as well as the optimized disposition of innovation resources also must be 
supported by the information infrastructure, therefore, the research of knowledge service platform has become a vital issue which need to be resolved urgently in the process of construction of innovational nation.

On the basis of the analysis of resources integration framework of the existing public service platform, we put forward the architecture of the public knowledge service platform based on the collaborative innovation, and analyze its concrete implementation. But we now only proposed the prototype design thought, on the foundation of collaboration scenario proposed in this article, In the next step of research work, we will further study on the realization details based on collaborative mechanism, such as: communication, data storage management, conflicts scheduling, and so on.

\section{Acknowledgments}

This paper is one of research results of project "The research on the institution of information service and the system of information guarantee in the construction of innovative nation" (number 06\&ZD031) funded by National Philosophy and Social Science foundation of China.

\section{References}

1. J. Howard, "Knowledge exchange networks in Australia's innovation system: overview and strategic analysis", A report to department of Education, Science and Training. Canberra: Howard Partners. 2005,6,pl-3

2. B. Kogut, and A. Turcanu, "The emergence of E-innovation: insights from open source software development", A working paper of the refined H. Jones Center, The Wharton School, University of Pennsylvania, 1999,p30

3. M. Sawhney, and E. Prandelli, "Communities of creation: managing distributed innovation in turbulent markets", California Management Review, summer, VOL.43, NO.4, pp.24-54, 2000

4. B. Wu, J.E. Chen, "innovation: a new innovation platform based on the internet", Engineering Management Conference, 2005. Proceedings 2005 IEEE International, Vol.2,p519-523

5. United States. Council on Competitiveness. 2004. Innovate America: Thriving in a World of Challenge and Change, National Innovative Initiative Report. 2004, p2-26.

6. National Science and Technology Library (April 20, 2007); http://www.nstl.gov.cn/index.html

7. http://www.calis.edu.cn/calisnew/

8. National Library of China (April 20, 2007);http://www.nlc.gov.cn

9. National science Library, Chinese Academy of Sciences (April 20, 2007); http://www.las.ac.cn/

10. Hubei Science Technology Library (April 20, 2007); http://www.hbstl.org.cn/index.jsp

11. Y.E. Yu, Y. Shi, W.Z. LI, "Research of SOA and Its System Building", Application Research of Computers, 2, p32-34(2005). 
12. Q. Chen, J. Shen, Y.Q. Dong, "Building a collaborative Manufacturing System on an Extensible SOA-based Platform", Proceedings of the 10th International Conference on Computer Supported Cooperative Work in Design, p1-6(2006). 